\title{
Mixed 20-peptide cancer vaccine in combination with docetaxel and dexamethasone for castration-resistant prostate cancer: a randomized phase II trial
}

\author{
Masanori Noguchi ${ }^{1,2}(1) \cdot$ Gaku Arai $^{5} \cdot$ Shin Egawa $^{6} \cdot$ Chikara Ohyama $^{7} \cdot$ Seiji Naito $^{8} \cdot$ Kazumasa Matsumoto $^{9}$. \\ Hirotsugu Uemura ${ }^{10}$. Masayuki Nakagawa ${ }^{11}$. Yasutomo Nasu ${ }^{12}$. Masatoshi Eto ${ }^{13}$. Shigetaka Suekane ${ }^{2}$. \\ Tetsuro Sasada ${ }^{14} \cdot$ Shigeki Shichijo $^{1} \cdot$ Akira Yamada $^{3} \cdot$ Tatsuyuki Kakuma $^{4} \cdot$ Kyogo Itoh $^{1}$
}

Received: 21 January 2019 / Accepted: 21 January 2020 / Published online: 5 February 2020

(c) The Author(s) 2020

\begin{abstract}
A novel cancer vaccine consisting of 20 mixed peptides (KRM-20) was designed to induce cytotoxic T lymphocytes (CTL) against twelve different tumor-associated antigens. The aim of this phase II trial was to examine whether KRM-20 in combination with docetaxel and dexamethasone enhances the antitumor effects in patients with castration-resistant prostate cancer (CRPC). In this double-blind, placebo-controlled, randomized phase II study, we enrolled chemotherapy-naïve patients with CRPC from ten medical centers in Japan. Eligible patients were randomly assigned 1:1 centrally to receive either KRM-20 combined with docetaxel and dexamethasone $(n=25)$ or placebo with docetaxel and dexamethasone $(n=26)$. The primary endpoint was the difference in prostate-specific antigen (PSA) decline between each treatment. The rates of $>50 \%$ PSA decline in the two arms were similar $(56.5 \%$ versus $53.8 \%$; $P=0.851)$. Human leukocyte antigen (HLA)-matched peptidespecific immunoglobulin G $(P=0.018)$ and CTL $(P=0.007)$ responses in the KRM-20 arm significantly increased after treatment. The addition of KRM-20 did not increase toxicity. There were no between-group differences in progression-free or overall survival (OS). The addition of KRM-20 was safe, and similar PSA decline and HLA-matched peptide-specific CTL and IgG responses increased in combination with docetaxel and dexamethasone in CRPC patients. Subgroup analysis suggested that this treatment is favorable for CRPC patients with $\geq 26 \%$ lymphocytes or PSA levels of $<11.2 \mathrm{ng} / \mathrm{ml}$, but further clinical trials comparing $\mathrm{OS}$ are required.
\end{abstract}

Keywords Prostate cancer $\cdot$ Multiple-peptide vaccine $\cdot$ Immunotherapy $\cdot$ Docetaxel $\cdot$ Phase II trial

\section{Abbreviations \\ CRPC Castration-resistant prostate cancer \\ CTL Cytotoxic T lymphocytes \\ ECOG Eastern Cooperative Oncology Group}

Previous publication: The study has been previously presented as an abstract and poster at the 2018 Genitourinary Cancers Symposium, February 8-10, 2018, San Francisco, CA, USA [1]. All figures and tables in this manuscript are new, that they were not published before in a similar or the same way in another article.

\section{Electronic supplementary material The online version of this} article (https://doi.org/10.1007/s00262-020-02498-8) contains supplementary material, which is available to authorized users.

Masanori Noguchi

noguchi@med.kurume-u.ac.jp

Extended author information available on the last page of the article

$\begin{array}{ll}\text { HLA } & \text { Human leukocyte antigen } \\ \text { IgG } & \text { Immunoglobulin G } \\ \text { MDSC } & \text { Myeloid-derived suppressor cells } \\ \text { OS } & \text { Overall survival } \\ \text { PBMC } & \text { Peripheral blood mononuclear cells } \\ \text { PD } & \text { Progressive disease } \\ \text { PFS } & \text { Progression-free survival } \\ \text { PSA } & \text { Prostate-specific antigen } \\ \text { TAA } & \text { Tumor-associated antigen } \\ \text { Treg } & \text { Regulatory T cells }\end{array}$

\section{Introduction}

Prostate cancer-related death is common among patients with metastatic castration-resistant prostate cancer (CRPC) in which the disease progresses despite androgen deprivation therapy. Although several new agents for CRPC, such as 
sipuleucel-T, cabazitaxel, abiraterone acetate, enzalutamide, and radium-223, have been approved recently based on their demonstrated overall survival (OS) benefits in phase 3 studies, each of these prolonged survival by only a few months [2-6]. Thus, there remains a need for treatments that can provide stable disease control and long-term survival benefits. After the approval of sipuleucel-T for patients with asymptomatic or minimally symptomatic CRPC based on survival benefits [2], and stable responses observed with the checkpoint inhibitors ipilimumab and nivolumub in other malignancies [7-9], immunotherapy has emerged as a viable and attractive strategy for the treatment of CRPC. However, immunotherapy alone may be unable to induce an immune response of sufficient potency to result in tumor regression when immune tolerance and large tumor burden are present.

The standard first-line chemotherapy for patients with progressive CRPC has consisted of docetaxel and oral prednisone $[10,11]$. Several animal studies have examined the use of cancer vaccines in combination with docetaxel, and reported enhancement of T-cell responses with antitumor activity [12-14]. These studies suggested that docetaxel is able to reduce the tumor burden and immune-suppressing elements, such as the increase in myeloid-derived suppressor cells (MDSC) in the tumor microenvironment, and it is well known that tumor-associated immunosuppression plays a significant role in tumor progression and resistance to immunotherapy [13].

We developed a novel cancer vaccine consisting of 20 mixed peptides (KRM-20) for patients with CRPC designed to induce cytotoxic T lymphocytes (CTL) against twelve different tumor-associated antigens (TAAs) highly expressed in prostate cancer tissues. The CTL epitopes represented by these 20 peptides are restricted to human leukocyte antigen (HLA)-A2, -A24, -A3 super type (-A3, -A11, -A31 or -A33) or -A26 of major histocompatibility complex class 1 molecules, providing coverage of the majority of patients who have different HLA alleles. A previous phase I study on KRM-20 for patients with CRPC demonstrated the feasibility, safety, and rapid and high immune responses without changes in immunosuppressive cell subsets [15].

This phase II study was designed to evaluate if docetaxel has the ability to alter components of the immune system independent of antitumor activity, and to examine the potential synergistic activity of the 20-mixed peptides vaccine in combination with docetaxel and dexamethasone.

\section{Patients and methods}

\section{Patient population}

For this phase II, randomized, double-blind, placebo-controlled study, we enrolled chemotherapy-naïve patients with CRPC from ten medical centers in Japan. The subjects must satisfy the following conditions: (1) patients must be diagnosed as prostate cancer pathologically at the initial treatment; (2) patients who had progressive disease after androgen deprivation therapy (ADT) either by surgical castration, gonadotropin-releasing hormone or antagonist treatment. Progressive disease while receiving ADT, defined by any 1 of the following: (1) at least two consecutive rises in serum PSA obtained at a minimum of 1-week intervals; (2) measurable disease with $\geq 50 \%$ increase in the sum of the cross products of all measurable lesions, or the development of new measurable lesions by RESIST; (3) non-measurable (bone) disease consisting of new areas of uptake by bone scan consistent with metastatic disease compared to previous imaging; (4) patients have serum PSA level $\geq 2 \mathrm{ng} / \mathrm{m}$; (5) anti-androgen therapy is discontinued for at least 4 weeks before the first vaccination for patients receiving flutamide and 6 weeks for those receiving bicalutamide; (6) patients continue to stay on medical treatment such as LHRH agonists or LHRH antagonists to maintain testosterone level of $0.5 \mathrm{ng} / \mathrm{m}$; (7) patients must be positive for HLA-A2, HLA-A24, HLA-A26 or HLA-A3 super type (A3, A11, A31, A33); (8) written informed consent must be obtained from patients; (9) patients must be more 20 year-old; (10) patients must be at a score level of 0 or 1 of an Eastern Cooperative Oncology Group (ECOG) performance status; (11) patients must be expected to survive more than 6 months; (12) patients must satisfy bone marrow function (white blood cell count $\geq 2500 / \mathrm{mm}^{3}$, lymphocyte count $\geq 1000 / \mathrm{mm}^{3}$, hemoglobin $\geq 8 \mathrm{~g} / \mathrm{dl}$, and platelets $\geq 100,000 / \mathrm{mm}^{3}$ ), hepatic function [total bilirubin $\leq 1.5 \times$ the upper limit of normal (ULM), transaminase $\leq 2 \times \mathrm{ULM}$ ], and renal function (serum creatinine $\leq 2 \times \mathrm{ULM}$ ). Patients without previous bilateral orchiectomy continued receiving luteinizing hormone-releasing agonists. Exclusion criteria included acute infection, history of severe allergic reactions, pulmonary, cardiac or other systemic diseases, or other inappropriate conditions for enrollment as judged by clinicians.

\section{Study design and treatment}

Randomization was performed centrally at the clinical research unit of Kurume University in Kurume, Japan. Patients were randomly assigned in a 1:1 ratio to receive either KRM-20 (study arm) or placebo (control arm) followed by intravenous docetaxel using a minimization technique with the following stratification factors: age ( $<65$ or $\geq 65$ years old) and PSA ( $<20$ or $\geq 20 \mathrm{ng} / \mathrm{ml})$. This study was double-blinded, and all physicians, patients, and investigators giving the interventions, assessing outcomes, and analyzing data were blinded to treatment assignment. In the event of a medical emergency in an individual patient, the 
treating physician was informed of the assigned treatment by a judge from the safety committee.

KRM-20 was designed to induce CTL against 20 peptides originating from twelve different TAAs, including PSA, prostatic acid phosphatase (PAP), prostate-specific membrane antigen (PSMA), epidermal growth factor-receptor (EGF$\mathrm{R})$, parathyroid hormone-related peptide (PTHrP), squamous cell carcinoma antigens 3 (SART3), cyclophilin B (CypB), Wolf-Hirshhorn syndrome critical region 2 (WHSC2), ubiquitin-conjugated enzyme variant Kua (UBE2V), heterogeneous nuclear ribonucleoprotein L (HNRPL), p56 ${ }^{l c k}$, and multidrug resistance-associated protein 3 (MRP3), as reported previously [15]. The name, source TAA, position, amino acid sequence, and HLA type of the KRM-20 including the 20 peptides are shown in Supplementary Table 1.

Patients received either KRM-20 $(20 \mathrm{mg} / 0.5 \mathrm{ml})$ or placebo $(0.5 \mathrm{ml})$ mixed with incomplete Freund's adjuvant (Montanide ISA-51VG; Seppic, Paris, France) subcutaneously on days $1,8,15,22$, and 29 with oral dexamethasone (1 mg) once daily on days 1-36. On day 36 , one hour after intravenous docetaxel at $70 \mathrm{mg} / \mathrm{m}^{2}$, patients received subcutaneous KRM-20 or placebo injection. Treatment with docetaxel and the study drug was repeated every 3 weeks for up to five cycles, and patients continued oral dexamethasone once daily until the end of the study. Dosing delay and reduction for docetaxel was permitted if toxic effects were noted. It was possible to hold docetaxel for less than 2 weeks until recovery, or reduce it to 60 or $50 \mathrm{mg} / \mathrm{m}^{2}$ in the event of neutropenia $\left(<2000 / \mathrm{mm}^{3}\right)$, platelets $<100,000 / \mathrm{mm}^{3}$, hemoglobin $<8 \mathrm{~g} / \mathrm{dl}$, total bilirubin $>1.5 \times \mathrm{ULM}$, transaminase $>2 \times$ ULM, or serum creatinine $>2 \times$ ULM. If docetaxel was held for more than 3 weeks, the patient was removed from protocol treatment. Patients who received protocol treatment were followed-up for 3 years for survival analyses.

\section{Outcomes}

For the primary endpoint of PSA decline, patients were evaluated at pre-treatment and every docetaxel cycle using serum PSA concentrations (at pre-treatment, and sixth to tenth and 3 weeks after protocol treatment). The rate of $>50 \%$ PSA decline was compared between arms. Patients were evaluated at pre-treatment and 3 weeks after protocol treatment by bone scans and computed tomography (CT) scans of the abdomen and pelvis followed by a 6 -month interval. The secondary endpoints included immune responses, safety profile, progression-free survival (PFS), and OS. To assess immune responses during the protocol treatment, peripheral blood was collected at pre-treatment, and sixth, eighth, and tenth study drug injections. HLA-matched peptide-specific immunoglobulin $\mathrm{G}(\mathrm{IgG})$ titer in the plasma were measured using a Luminex system [16], and HLA-matched peptidespecific CTL in peripheral blood mononuclear cells (PBMC) was evaluated by IFN- $\gamma$ ELISPOT assay, as described previously [15]. When the total HLA-matched peptide-specific IgG titers at the tenth study drug injection was higher than that at pre-vaccination, it was considered to be a positive response. Positive CTL responses were defined as a greater than 100-spot increase in the total number of HLA-matched peptide-specific IFN- $\gamma$ spots at the tenth study drug injection. We also measured Treg and MDSC at the same points for exploratory analysis of immune suppression. Treg were defined as CD4+ CD25+ FoxP3+ cells among lymphocytes, and MDSC were identified as CD33+ 11b+ cells from the lineage markers (CD3, CD19, CD56, and CD16)- and HLADR-cells measured by multicolor flow cytometry. The safety profile was assessed throughout the study by monitoring for adverse events (AEs) [according to the National Cancer Institute Common Terminology Criteria for Adverse Events version 4.0 (NCI-CTC Ver. 4)], chemical laboratory tests, vital signs, and physical examinations. PFS was defined as the time in months from randomization until objective disease progression based on the PSA Working Group Consensus Criteria 2 (PCWG2) [17], the Response Evaluation Criteria in Solid Tumors (RECIST) $1 \cdot 1$ criteria, or death. OS was calculated as the time in months from the date of randomization to death or to the date of last contact for censored observations. Analyses of primary and secondary efficacy endpoints were based on the intention-to-treat (ITT) population that included all randomly assigned patients.

\section{Statistical design and analysis}

The primary endpoint of this study was the comparison of each treatment arm for the rate of $>50 \%$ PSA decline from baseline. Based on the previous report [18], the assumed rate of $>50 \%$ PSA decline was $65 \%$ in the KRM-20 arm and $25 \%$ in the placebo arm. The target sample size was 50 assuming an ineligibility rate of approximately $10 \%$. Sample size computation based on the large sample test was performed with the following assumptions: type I error rate $=0.05$, power $80 \%$ and the ratio of the two groups as 1:1. The Student's $t$ test and chi-square test were used to compare quantitative and categorical variables among safety profiles and immune responses to the treatment, respectively. PFS and OS data for each arm were analyzed using the Kaplan-Meier method. The log-rank test was used for the comparison of the survival curves, and Cox proportional hazard analysis was used to estimate hazard ratios (HR). The confidence intervals (CI) reported were 95\%. Cox proportional hazards regression model was used for univariate and multivariate analyses to identify factors that significantly impacted survival. All baseline parameters in the survival and proportional hazards regression analysis were analyzed as dichotomous variables using median or cut-off values. Statistical analyses were 
performed using SAS software version 9.1 (SAS Institute, Cary, NC, USA) with a two-sided significance level of $5 \%$.

\section{Results}

Between July 31, 2013 and July 11, 2014, 55 chemotherapy-naive patients with progressive CRPC were screened for enrollment at ten medical centers in Japan (Fig. 1). Fifty-one patients were enrolled and randomly assigned to receive either KRM-20 with docetaxel and dexamethasone $(n=25)$ or placebo $(n=26)$ with docetaxel and dexamethasone. Two patients in the KRM-20 arm were not treated due to the physician's decision or death, and not included in the safety analysis set. Baseline demographic and clinical characteristics of participants, including median age, ECOG performance status, metastatic site, HLA types, Gleason scores, median PSA levels, median times from diagnosis to study entry, median IgG levels, and median CTL levels were balanced between the two arms (Table 1). Most patients (72\% in the KRM-20 arm and $69.2 \%$ in the placebo arm) had bone disease, and all patients were refractory to previous hormone therapies. At the end of study treatment, 17 (73.9\%) of 23 patients in the KRM-20 arm and $20(76.9 \%)$ of 26 patients in the placebo arm had completed the study treatment (Fig. 1).

The rates of $>50 \%$ PSA decline in the two arms were similar (56.5\% vs. $53.8 \%)$, with no significant difference ( $P=0.851$, chi-square test).

Regarding immune responses, the mean total HLAmatched peptide-specific IgG $(P=0.014, t$ test $)$ and CTL $(P=0.007, t$ test) responses in the KRM-20 arm significantly
Fig. 1 Study flowchart. DTX dexamethasone

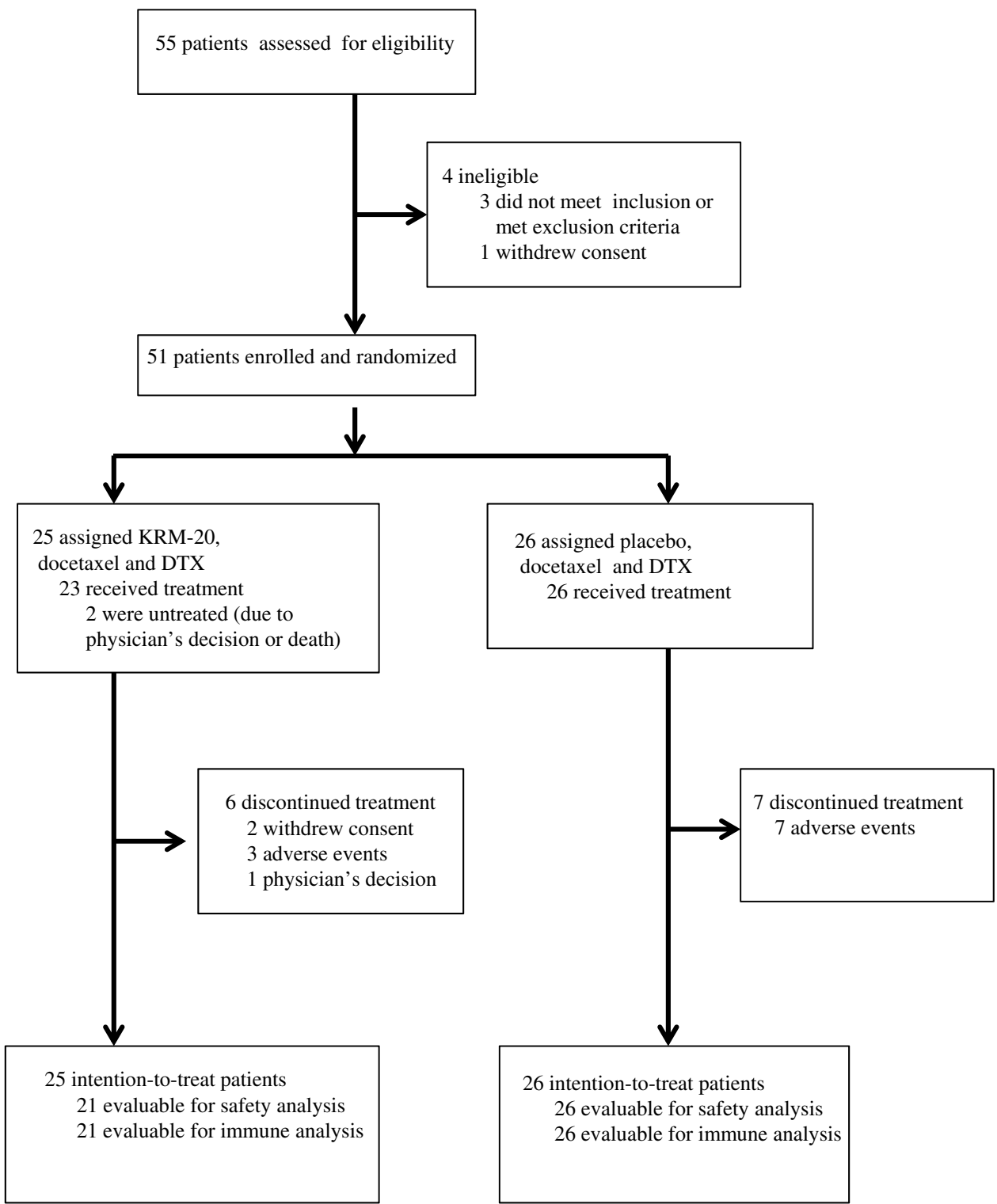


Table 1 Patient demographics and baseline characteristics

\begin{tabular}{|c|c|c|c|}
\hline & KRM-20 arm $(n=25)$ & Placebo arm $(n=26)$ & Total $(n=51)$ \\
\hline \multicolumn{4}{|l|}{ Age (years) } \\
\hline Median & 72 & 70 & 71 \\
\hline Range & $41-85$ & $51-81$ & $41-85$ \\
\hline \multicolumn{4}{|c|}{ ECOG performance status } \\
\hline 0 & $22(88 \%)$ & $23(88.5 \%)$ & $45(88 \%)$ \\
\hline 1 & $3(12 \%)$ & $3(11.5 \%)$ & $6(12 \%)$ \\
\hline \multicolumn{4}{|c|}{ Time from diagnosis to study entry (years) } \\
\hline Median & 2 & 4 & 4 \\
\hline Range & $0.6-12$ & $0.8-9$ & $0.6-12$ \\
\hline \multicolumn{4}{|c|}{ Gleason score at diagnosis } \\
\hline$<8$ & $4(16 \%)$ & $5(19 \%)$ & $9(18 \%)$ \\
\hline$\geq 8$ & $19(76 \%)$ & $21(81 \%)$ & $40(78 \%)$ \\
\hline Unknown & $2(8 \%)$ & 0 & $2(4 \%)$ \\
\hline \multicolumn{4}{|l|}{ HLA type } \\
\hline $\mathrm{A} 24$ & $18(72 \%)$ & $17(65.4 \%)$ & $35(67 \%)$ \\
\hline $\mathrm{A} 2$ & $10(40 \%)$ & $10(38.5 \%)$ & $20(39 \%)$ \\
\hline A26 & $6(24 \%)$ & $5(19.2 \%)$ & $11(22 \%)$ \\
\hline A3 family & $13(52 \%)$ & $13(50 \%)$ & $26(51 \%)$ \\
\hline \multicolumn{4}{|l|}{ Metastatic sites } \\
\hline None & $1(4 \%)$ & $4(15.4 \%)$ & $5(10 \%)$ \\
\hline Bone & $18(72 \%)$ & $18(69.2 \%)$ & $36(71 \%)$ \\
\hline Lymph node & $12(48 \%)$ & $12(46.2 \%)$ & $24(47 \%)$ \\
\hline Lung & $2(8 \%)$ & 0 & $2(4 \%)$ \\
\hline Liver & $1(4 \%)$ & 0 & $1(2 \%)$ \\
\hline Others & $11(44 \%)$ & $8(30.8 \%)$ & $19(37 \%)$ \\
\hline Unknown & $2(8 \%)$ & 0 & $2(4 \%)$ \\
\hline \multicolumn{4}{|l|}{ Previous treatment } \\
\hline Hormone therapy & $25(100 \%)$ & $26(100 \%)$ & $51(100 \%)$ \\
\hline Prostatectomy & $4(16 \%)$ & $3(11.5 \%)$ & $7(14 \%)$ \\
\hline Radiation & $9(36 \%)$ & $6(23.1 \%)$ & $15(29 \%)$ \\
\hline \multicolumn{4}{|l|}{ PSA (ng/ml) } \\
\hline Median & 11.2 & 10.1 & 11.2 \\
\hline Range & $3.7-663.3$ & $2.4-299.9$ & $2.4-663.3$ \\
\hline \multicolumn{4}{|l|}{ Neutrophils, $\%$} \\
\hline Median & 63.8 & 67.4 & 65.0 \\
\hline Range & $49.8-89$ & $42.5-82.2$ & $42.5-89$ \\
\hline \multicolumn{4}{|l|}{ Lymphocytes, \% } \\
\hline Median & 27.5 & 24.9 & 26.0 \\
\hline Range & $6.3-42$ & $13.3-49.4$ & $6.3-49.4$ \\
\hline \multicolumn{4}{|l|}{ IgG, FIU } \\
\hline Median & 1053 & 1282.5 & 1142 \\
\hline Range & $519-57,231$ & $136-92,009$ & $136-92,009$ \\
\hline \multicolumn{4}{|l|}{ CTL, spots } \\
\hline Median & 13 & 1 & 4 \\
\hline Range & $0-280$ & $0-53$ & $0-280$ \\
\hline \multicolumn{4}{|l|}{ MDSC, $\%$} \\
\hline Median & 9.6 & 9.4 & 9.6 \\
\hline Range & $1.3-22$ & $0.1-20.8$ & $0.1-22$ \\
\hline \multicolumn{4}{|l|}{ Treg, \% } \\
\hline Median & 2.7 & 3.2 & 2.8 \\
\hline Range & $0.9-9.4$ & $0.8-6.2$ & $0.8-9.4$ \\
\hline
\end{tabular}

CTL, cytotoxic T lymphocytes; ECOG, Eastern Cooperative Oncology Group; FIU, fluorescence intensity units; HLA, human leukocyte antigen; IgG, immunoglobulin G; MDSC, myeloid-derived suppressor cells; Treg, regulatory $\mathrm{T}$ cells 
increased after treatment, whereas the IgG and CTL responses in the placebo arm did not increase after treatment (Fig. 2a, b). The median number of HLA-matched peptides in the KRM-20 arm was 16 (8-17), and peptide-specific IgG and CTL responses matching HLA were observed in $8(35 \%)$ of 23 patients and 5 (22\%) of 23 patients, respectively (Supplementary Table 2). In the exploratory analysis for immune suppression, the numbers of both Treg and MDSC among PBMC in the two arms did not increase during treatment (Fig. 2c, d), and the number of MDSC in the KRM-20 arm significantly decreased after the treatment $(P=0.03, t$ test $)$ (Fig. 2d).

AEs in the two arms during treatment are summarized in Supplementary Table 3. The most common AEs (occurring in more than $40 \%$ patients in one or both arms) were injection site reactions, alopecia, neutropenia, and peripheral neuropathy. AEs of grade 3 or higher developed in similar frequencies between the two arms: $16(70 \%)$ of 23 patients in the KRM-20 arm and 18 (79\%) of 26 patients in the placebo

IgG

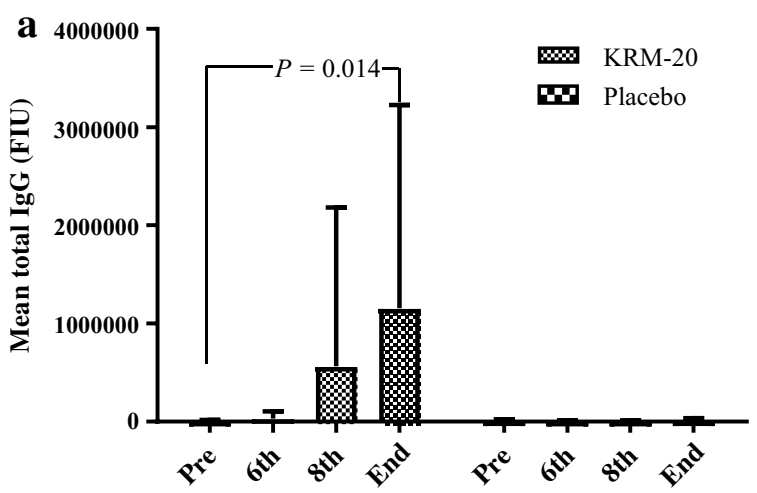

c

MDSC

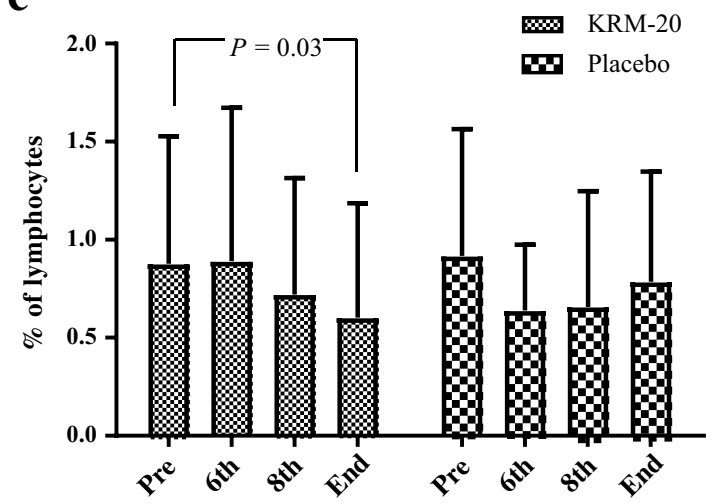

Fig. 2 Immune responses in patients during treatment. a IgG responses in the KRM-20 arm significantly increased after treatment $(P=0.014, t$ test). b CTL responses in the KRM-20 arm significantly increased after treatment $(P=0.007, t$ test). $\mathbf{c}$ The number of MDSC in the KRM-20 arm significantly decreased after treatment $(P=0.03$, arm. Grade 5 events during treatment were observed in 2 patients with pneumonia in the placebo arm. The addition of KRM-20 did not increase toxicity. The dose of docetaxel was reduced due to hematological toxicity in a similar proportion in each patient arm (17.5\% in the KRM-20 arm and $15 \%$ in the placebo arm).

After the median follow-up of 8.3 months (IQR 5.0-13.3), $45(88 \%)$ of 51 patients had disease progression or died: 22 $(88 \%)$ in the KRM-20 and $23(85 \%)$ in the placebo arm. Based on investigator assessment of disease response and progression using PCWG2 or RECIST criteria, partial response was observed in $2(8 \%)$ patients in the KRM-20 arm and $3(12 \%)$ patients in the placebo arm. No complete responses were observed in either arm. The median PFS time was 8.9 months (95\% CI 4.9-12.2) in the KRM20 arm and 7.4 months (95\% CI 5.3-12.5) in the placebo arm (Fig. 3a), but this difference was not significant (HR $1.0 ; 95 \%$ CI $0.6-1.9 ; P=0.87$ ). At the data cut-off date of July 1, 2017 after a median follow-up of 32.4 months (IQR

\section{CTL}
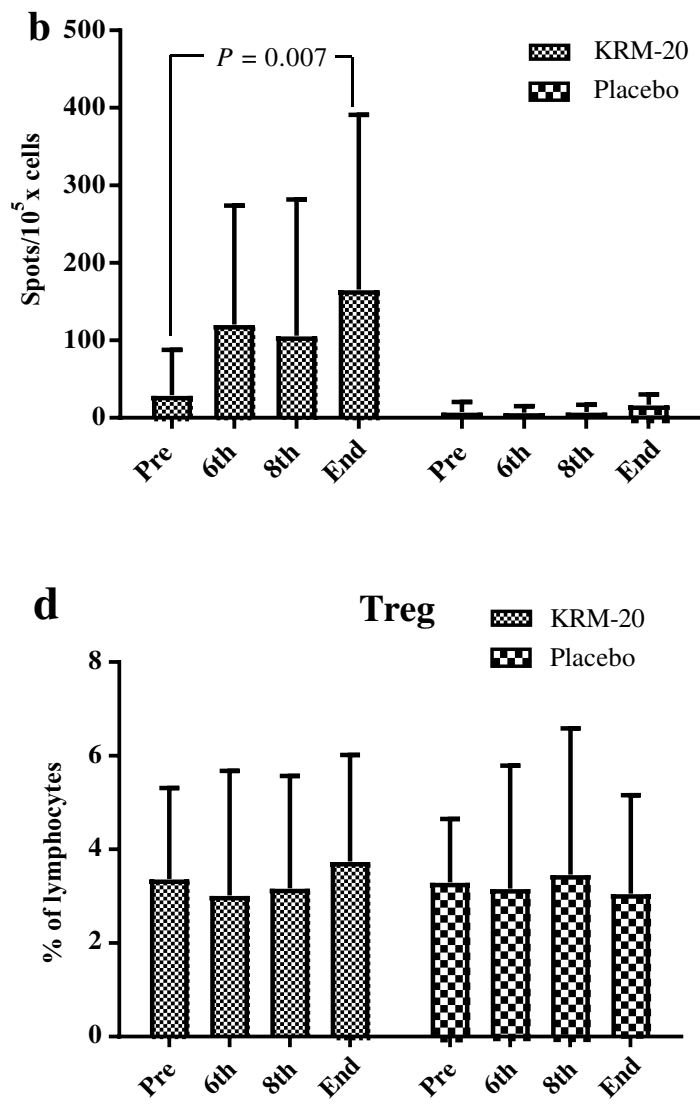

$t$ test). d The number of Treg in PBMC in both arms did not increase during treatment. $C T L$ cytotoxic T lymphocytes, $I g G$ immunoglobulin G, $M D S C$ myeloid-derived suppressor cells, $P S A$ prostate-specific antigen, Treg regulatory $\mathrm{T}$ cells 

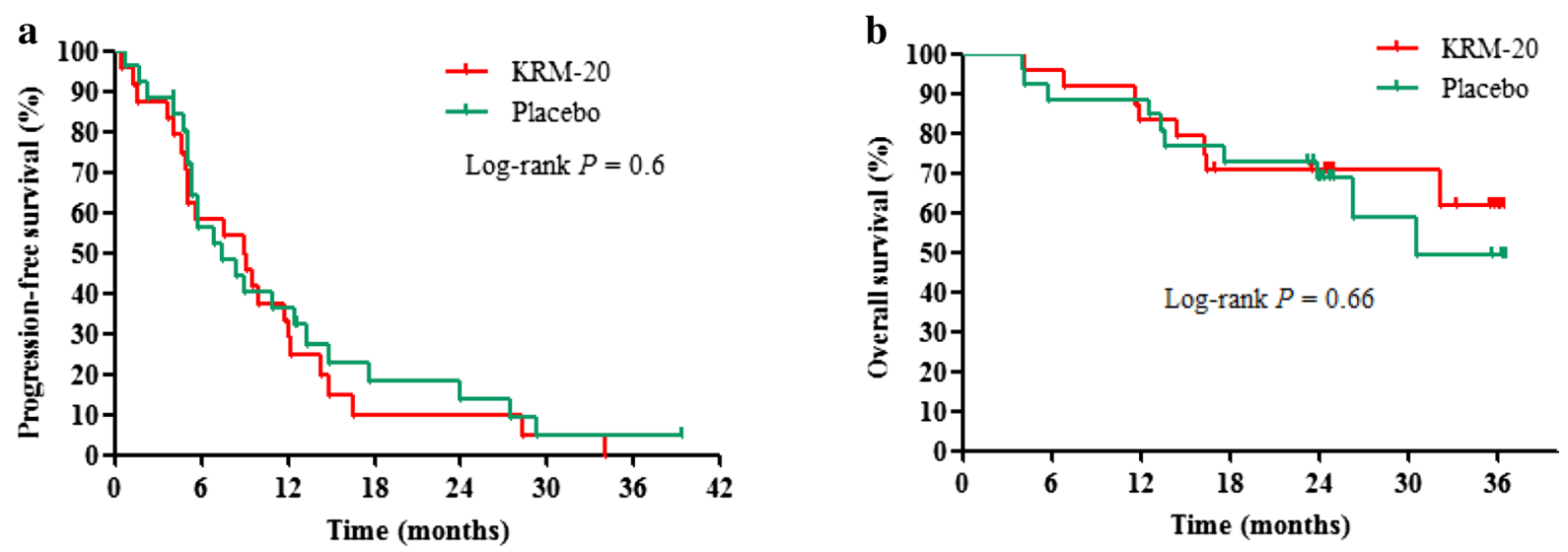

$\begin{array}{cccccccc}\text { No at risk } & & & & & & & \\ \text { KRM-20 } & 25 & 15 & 8 & 3 & 3 & 2 & 0 \\ \text { Placebo } & 26 & 15 & 10 & 5 & 4 & 2 & 2\end{array}$
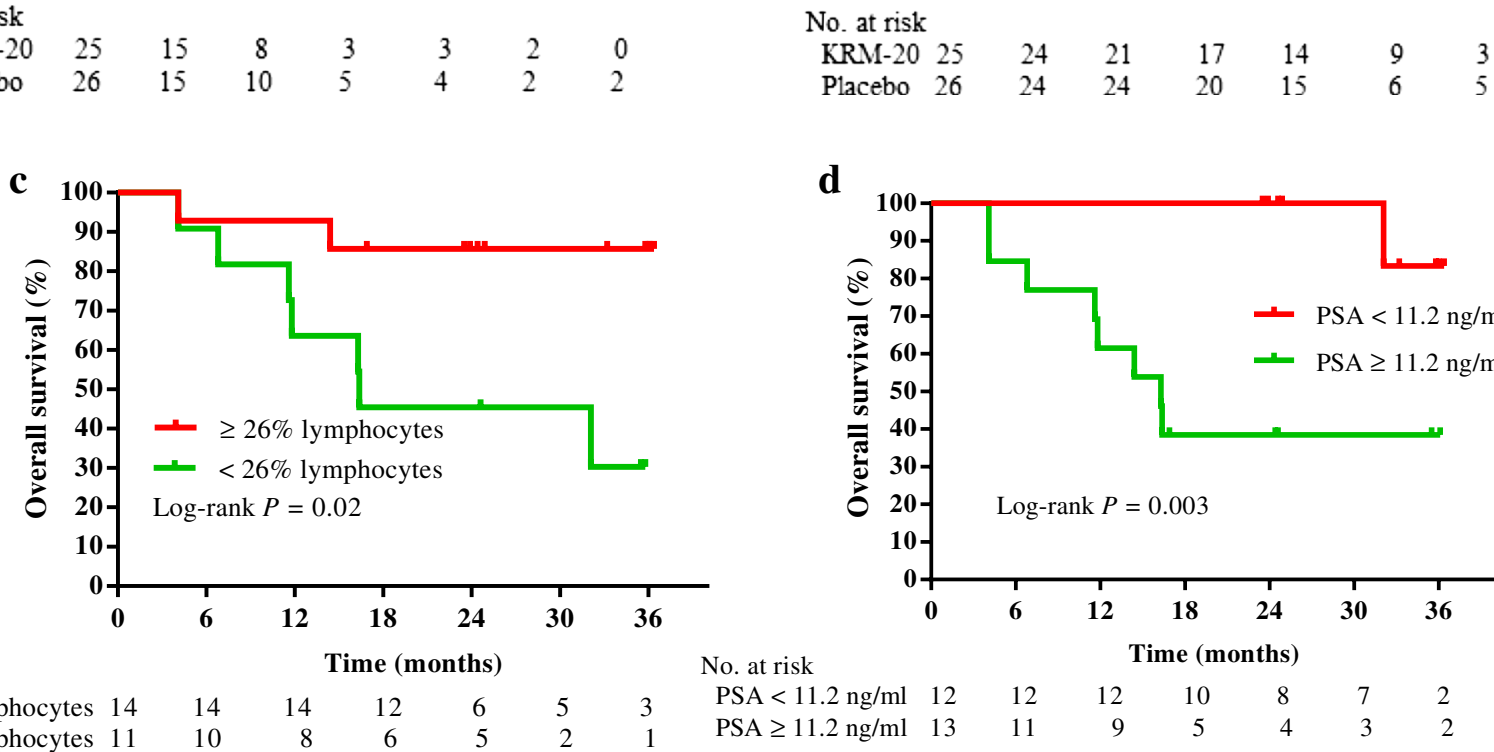

No. at risk

$\begin{array}{lrrrrrrr}\geq 26 \% \text { lymphocytes } & 14 & 14 & 14 & 12 & 6 & 5 & 3 \\ <26 \% \text { lymphocytes } & 11 & 10 & 8 & 6 & 5 & 2 & 1\end{array}$

d

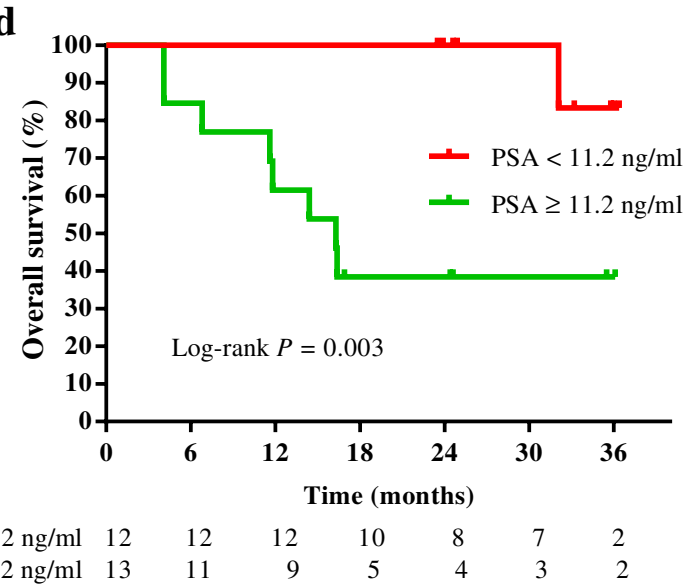

Fig. 3 Kaplan-Meier curves. Progression-free survival (a) and overall survival (b) in the intention-to-treat population. Overall survival in KRM20 patients according to cut-offs of $26 \%$ lymphocytes (c) or PSA level of $11.2 \mathrm{ng} / \mathrm{ml}$ (d). PSA prostate-specific antigen

16.3-36.1), 24 (47\%) patients in the ITT population had died: 12 (48\%) patients in the KRM-20 arm and 12 (46\%) patients in the placebo arm. The median OS time in the placebo arm was 30.5 months (95\% CI 23.9-38.8), but it has yet to be reached in the KRM-20 arm (95\% CI 16.4not reached); it was estimated to be 37.7 months using the adjusted HR (Fig. 3b). Although patients in the KRM-20 arm had a slightly longer OS than those in the placebo arm, there was no between-group difference in median OS time (HR 0.81; 95\% CI 0.3-2.1; $P=0.83$ ). To further investigate the effects of KRM-20 with docetaxel and dexamethasone, Cox proportional hazards regression analysis was performed to find factors that can predict disease response in the KRM-20 arm. Univariate Cox analysis demonstrated \% lymphocytes $(P=0.006)$ and PSA level $(P=0.003)$ to be significantly associated with survival. The factors with an HR less than 0.5 in the univariate analysis were included in the multivariate analysis of the model. In the KRM-20, $\geq 26 \%$ lymphocytes (HR 0.3; 95\% CI 0.08-0.93; $P=0.04$ ) and PSA levels $<11.2 \mathrm{ng} / \mathrm{ml}$ (HR 0.15; 95\% CI 0.03-0.58; $P=0.004$ ) were found to be significantly favorable factors for OS (Table 2). Consequently, the median OS of patients with $\geq 26 \%$ lymphocytes or PSA levels $<11.2 \mathrm{ng} / \mathrm{ml}$ was significantly longer than that in their counterparts in the KRM20 (median OS, not reached vs 16.4 months; $P=0.02$ or median OS, not reached vs 16.3 months; $P=0.003$, respectively; Fig. 3c, d).

\section{Discussion}

This randomized phase II trial of KRM-20 in combination with docetaxel and dexamethasone for patients with CRPC demonstrated a similar PSA decline, increased immune responses, and reduction in MDSC compared with docetaxel and dexamethasone treatment alone. PSA decline in several 
Table 2 Cox proportional hazards regression analysis of associations between potential factors and overall survival in the 25 CRPC patients

\begin{tabular}{|c|c|c|c|c|c|c|c|}
\hline \multirow[t]{2}{*}{ Factors } & \multirow[t]{2}{*}{ Cut-offs ${ }^{\mathrm{a}}$} & \multicolumn{3}{|c|}{ Univariate } & \multicolumn{3}{|c|}{ Multivariate } \\
\hline & & HR & $95 \%$ CI & $P$ value & HR & $95 \% \mathrm{CI}$ & $P$ value \\
\hline Lymphocytes, \% & $\geq 26$ vs $<26$ & 0.09 & $0.005-0.56$ & 0.006 & 0.30 & $0.08-0.93$ & 0.04 \\
\hline PSA, ng/ml & $<11.2$ vs $\geq 11.2$ & 0.22 & $0.06-0.60$ & 0.003 & 0.15 & $0.03-0.58$ & 0.004 \\
\hline Performance status & 0 vs 1 & 0.36 & $0.09-2.45$ & 0.25 & 0.51 & $0.15-2.32$ & 0.34 \\
\hline Gleason score & $<8$ vs $\geq 8$ & 0.45 & $0.10-3.05$ & 0.35 & 0.24 & $0.06-1.09$ & 0.06 \\
\hline Neutrophils, \% & $<65$ vs $\geq 65$ & 0.51 & $0.16-1.16$ & 0.1 & - & - & - \\
\hline Treg, $\%$ & $<2.8$ vs $\geq 2.8$ & 0.63 & $0.21-1.75$ & 0.38 & - & - & - \\
\hline MDSC, $\%$ & $<9.6$ vs $\geq 9.6$ & 0.72 & $0.25-2.02$ & 0.52 & - & - & - \\
\hline Age, years & $\geq 71 \mathrm{vs}<71$ & 0.75 & $0.19-3.11$ & 0.68 & - & - & - \\
\hline IgG, FIU & $\geq 1142$ vs $<1142$ & 0.79 & $0.31-2.03$ & 0.62 & - & - & - \\
\hline CTL, spots & $\geq 4$ vs $<4$ & 1.04 & $0.41-2.82$ & 0.94 & - & - & - \\
\hline
\end{tabular}

Of the 25 patients, 9 died

CTL, cytotoxic T lymphocytes; FIU, fluorescence intensity units; HR, hazard ratio; IgG, immunoglobulin G; MDSC, myeloid-derived suppressor cells; Treg, regulatory T cells

${ }^{\mathrm{a}} \mathrm{Cut}-\mathrm{offs}$ are based on median values clinical trials for CRPC have been associated with longer survival [19]. However, improvements in OS in patients with CRPC may be observed without differences in PSA response rates [10], and PSA decline is not considered a true response. These results suggest the difficulty in clinical assessment of the phase II study setting for CRPC.

Active specific immunotherapy using either TAAs or their peptides has failed to provide clinical benefits for cancer patients in the large number of clinical trials since the 1990s, and the mechanisms involved in the failure have not been clarified [20-22]. Furthermore, this study did not demonstrate a longer OS or PFS than with the placebo. To understand the mechanisms involved in the failure of KRM-20, we predicted subsets of patients who will respond to KRM-20 with good clinical effects. Subgroup analysis suggested that this treatment is favorable for CRPC patients with $\geq 26 \%$ lymphocytes or PSA levels $<11.2 \mathrm{ng} / \mathrm{ml}$. Although further validation is required, this finding is novel and helpful to prolong survival in patients with CRPC treated by peptide vaccinations.

In this study, assessment of HLA-matched peptidespecific IgG and CTL was conducted primarily to evaluate immune responses to the combination therapy with KRM20. A significant increase in both HLA-matched peptidespecific IgG and CTL responses was observed in patients receiving the combination of KRM-20 with docetaxel and dexamethasone compared with patients in the placebo arm. The administration of cytotoxic chemotherapy agents, such as docetaxel, induces bone marrow suppression, and has been believed to negatively affect immune responses induced by the cancer vaccine. However, recent data suggest that many widely used chemotherapeutic agents have beneficial immunomodulatory effects thorough several mechanisms, including cytokine production, T cell infiltration of tumors, and maturation of dendritic cells [23-25]. The dose and scheduling of docetaxel in combination with cancer vaccines remain unknown. In a murine model, the combination of repeated melanoma tumor cell vaccinations with a more clinically relevant dose of docetaxel did not impede T-cell activation [26]. A previous randomized phase II trial of a cancer vaccine in combination with the clinical dose of docetaxel for metastatic CRPC patients did not inhibit vaccine-specific $T$ cell reactivity $[27,28]$, and the present study demonstrated that KRM-20 in combination with docetaxel and dexamethasone increased HLA-matched peptidespecific IgG and CTL responses. Several clinical trials have suggested that cancer vaccines alter the clinical effects of subsequent docetaxel. An early phase trial of an adenovirusbased vaccine targeting p53 in patients with non-small cell lung cancer reported higher objective responses to salvage chemotherapy initiated after vaccine treatment [29]. Similarly, in a survival analysis of the pivotal randomized phase III IMPACT trial, patients who received sipuleucel-T prior to docetaxel demonstrated longer survival than patients receiving placebo prior to docetaxel $(P=0.03)$ [2]. However, although not exactly different from the current trial, two phase III trials (VITAL-1 and VITAL-2) of GVAX which was composed of two human prostate cell lines LNCaP and PC3 as antigen source, were failed to demonstrate survival benefit. VITAL-2 compared the combination of GVAX plus docetaxel with standard docetaxel and prednisone in men with symptomatic CRPC. The VITAL-2 study was terminated early due to increased deaths in the vaccine arm. The VITAL-1 was also terminated based on a result of less than a 30\% chance of meeting an improved survival end point. Regarding the GVAX failure, it has been pointed out that the lack of placebo, docetaxel dose, and timing are not taken into account [30]. 
Another concern is the immune suppression caused by the continuous administration of low-dose dexamethasone with the peptide-based cancer vaccine. However, recent clinical trials found that combination therapy of low-dose dexamethasone with a peptide-based vaccine induced positive CTL responses, and a longer PSA PFS and OS than the peptide-based vaccine alone [31, 32].

In the current study, the number of MDSC, but not Treg, in patients in the KRM-20 arm decreased after treatment. Immune inhibition caused by MDSC in cancer patients has been reported in a number of studies. The increase in circulating MDSC also correlates with PSA levels and tumor burden in patients with CRPC [33]. Several preclinical studies reported that docetaxel combined with a cancer vaccine depleted circulating or tumor-infiltrating MDSC with CTL responses in murine tumor models by direct alteration of MDSC signaling, phenotype, and function [12, 13, 34]. These findings suggest potential clinical benefits by addition of docetaxel to the current immunotherapy.

The limitation of the present study was that the similar PSA decline, increase in immune responses, and decrease of MDSC in the KRM-20 arm did not lead to a longer PFS or OS than those in the placebo arm. This may have been due to the small number of patients and study design not comparing the difference in survival between the two arms. Further randomized clinical trials with a study design where the primary endpoint is the comparison of survival between KRM20 in combination with or without docetaxel are needed.

The safety and tolerability were similar to the known profiles for docetaxel and peptide-based vaccines $[10,15]$. The most common AEs were grade 1 or 2 injection site reactions in both arms. The main cause of grade 3 or higher AEs was hematology toxicity due to docetaxel, and these AEs developed in almost $40 \%$ of the patients in each arm. The addition of KRM-20 in combination with docetaxel and dexamethasone was feasible without increased toxicity.

In conclusion, KRM-20 in combination with docetaxel and dexamethasone for patients with CRPC resulted in a similar PSA decline, increased immune responses, and reduced MDSC compared with docetaxel and dexamethasone treatment alone. Subgroup analysis suggested that this treatment is favorable for CRPC patients with $\geq 26 \%$ lymphocytes or PSA levels $<11.2 \mathrm{ng} / \mathrm{ml}$. Although patients in the KRM-20 arm had a slightly longer OS than those in the placebo arm, this study did not demonstrate any survival benefits. Further large-scale clinical trials comparing OS are required to confirm the clinical benefits of this treatment.

Acknowledgements We thank all patients, staff, and investigators at the participating hospitals. We thank Teruhito Itoh for scientific advice.

Author contributions $\mathrm{MN}$ and $\mathrm{KI}$ conceived, designed, and supervised the study. MN, GA, SE, CO, SN, KM, HU, MN, ME, and SS recruited patients and provided clinical samples. TS, SS, AY, and TK acquired and analyzed the data. MN, TK, and KI drafted the manuscript. All authors read and approved the final version of the paper.

Funding This study was supported by a Grant from the Ministry of Health, Labour and Welfare of Japan (ID 2014110358 to Masanori Noguchi).

\section{Compliance with ethical standards}

Conflict of interest Kyogo Itoh received research funding from Taiho Pharmaceutical Company. Akira Yamada is a part-time executive of BrightPath biotherapeutics Co. Ltd. and has stock. Seiji Naito has received honorarium from Sanofi. The remaining authors declare that there are no other conflicts of interest.

Ethical approval and ethical standards The protocol was approved by institutional review boards or ethical committees at all of the institutions: The Ethical Committee of Kurume University, approval number 213101; The Kitasato Institute, Clinical Research Review Board, approval number 2013002; Okayama University Hospital Ethics Committee, approval number 251001; Dokkyo Medical University Ethical Committee, approval number 1304; The Ethical Committee of Kumamoto University Hospital, approval number 2013701; The Jikei University Certified Review Board, approval number 25-20(2921); The Ethical Committee of Hirosaki University Hospital, approval number Ishi1; Kyushu University Certified Institutional Review Board, approval number 2013301; The Ethical Committee of Kagoshima University Hospital, approval number 2014102; and Kindai University Certified Institutional Review Board, approval number 2013005, and it was registered in the UMIN Clinical Trials Registry (UMIN000011028). The study was carried out in accordance with the Declaration of Helsinki and the International Conference on Harmonization of Good Clinical Practice guidelines, and it was conducted in an outpatient setting.

Informed consent Written-informed consent to participate in the clinical trial and to use their data for research and publication purpose was received from all individual participants before participating in the study.

Open Access This article is licensed under a Creative Commons Attribution 4.0 International License, which permits use, sharing, adaptation, distribution and reproduction in any medium or format, as long as you give appropriate credit to the original author(s) and the source, provide a link to the Creative Commons licence, and indicate if changes were made. The images or other third party material in this article are included in the article's Creative Commons licence, unless indicated otherwise in a credit line to the material. If material is not included in the article's Creative Commons licence and your intended use is not permitted by statutory regulation or exceeds the permitted use, you will need to obtain permission directly from the copyright holder. To view a copy of this licence, visit http://creativecommons.org/licenses/by/4.0/.

\section{References}

1. Noguchi M, Arai G, Egawa S et al (2018) Mixed 20-peptide cancer vaccine in combination with docetaxel and dexamethasone for castration-resistant prostate cancer: a randomized, double-blind, placebo-controlled, phase 2 trial. J Clin Oncol 36(6 suppl):214 214 (Abstract 214) 
2. Kantoff PW, Higano CS, Shore ND et al (2010) Sipuleucel-T immunotherapy for castration-resistant prostate cancer. N Engl J Med 363:411-422

3. de Bono JS, Oudard S, Ozguroglu M et al (2010) Prednisone plus cabazitaxel or mitoxantrone for metastatic castration-resistant prostate cancer progressing after docetaxel treatment: a randomized open-label trial. Lancet 376:1147-1154

4. de Bono JS, Logothetis CJ, Molina A et al (2011) Abiraterone and increased survival in metastatic prostate cancer. N Engl J Med 364:1995-2005

5. Scher HI, Fizazi K, Saad F et al (2012) Increased survival with enzalutamide in prostate cancer after chemotherapy. N Engl J Med 367:1187-1197

6. Parker C, Nilsson S, Heinrich D et al (2013) Alpha emitter radium-223 and survival in metastatic prostate cancer. N Engl J Med 369:213-223

7. Hodge FS, O'Day SJ, McDermott DF et al (2010) Improved survival with ipilimumab in patients with metastatic melanoma. $\mathrm{N}$ Engl J Med 363:711-723

8. Topalian SL, Hodge FS, Brahmer JR et al (2012) Safety, activity, and immune correlates of anti-PD-1 antibody in cancer. N Engl J Med 366:2443-2454

9. Hamid O, Robert C, Daud A et al (2012) Safety and tumor responses with lambrolizumab (anti-PD-1) in melanoma. N Engl J Med 369:134-144

10. Tannock IF, de Wit R, Berry WR et al (2004) Docetaxel plus prednisone or mitoxantrone plus prednisone for advanced prostate cancer. N Engl J Med 351:1502-1512

11. Petrylak DP, Tangen CM, Hussain MH et al (2004) Docetaxel and estramustine compared with mitoxantrone and prednisone for advanced refractory prostate cancer. N Engl J Med 351:1513-1520

12. Garnett CT, Schlom J, Hodge JW (2008) Combination of docetaxel and recombinant vaccine enhances T-cell responses and antitumor activity: effects of docetaxel on immune enhancement. Clin Cancer Res 14:3536-3544

13. Kodumudi KN, Woan K, Gilvary DL, Sahakian E, Wei S, Djeu JY (2010) A novel chemoimmunomodulating property of docetaxel: suppression of myeloid-derived suppressor cells in tumor bearers. Clin Cancer Res 16:4583-4594

14. Hodge JW, Garnett CT, Farsaci B et al (2013) Chemotherapyinduced immunogenic modulation of tumor cells enhance killing by cytotoxic $\mathrm{T}$ lymphocytes and is distinct from immunogenic cell death. Int J Cancer 133:624-636

15. Noguchi M, Arai G, Matsumoto K et al (2015) Phase I trial of a cancer vaccine consisting of 20 mixed peptides in patients with castration-resistant prostate cancer: dose-related immune boosting and suppression. Cancer Immunol Immunother 64:493-505

16. Komatsu N, Shichijo S, Nakagawa M et al (2004) New multiplexed flow cytometric assay to measure antipeptide antibody: a novel tool for monitoring immune responses to peptides used for immunization. Scand J Clin Lab Investig 64:535-545

17. Scher HI, Halabi S, Tannock I et al (2008) Design and end points of clinical trials for patients with progressive prostate cancer and castrate levels of testosterone: recommendations of the Prostate Cancer Clinical Trials Working Group. J Clin Oncol 26:1148-1159

18. Picus J, Halabi S, Kelly WK et al (2011) A phase II study of estramustine, docetaxel, and bevacizumab in men with castration resistant prostate cancer: results of cancer and leukemia group B (CALGB) 90006. Cancer 117:526-533

19. Petrylak DP, Ankerst DP, Jiang CS et al (2006) Evaluation of prostate-specific antigen declines for surrogacy in patients treated on SWOG 99-16. J Natl Cancer Inst 98:516-521

20. Rosenberg SA, Yang JC, Schwartzentruber DJ et al (1998) Immunologic and therapeutic evaluation of synthetic peptide vaccine for the treatment of patients with metastatic melanoma. Nat Med $4: 321-327$

21. Schwartzentruber DJ, Lawson DH, Richards JM et al (2011) Gp100 peptide vaccine and interleukin-2 in patients with advanced melanoma. N Engl J Med 364:2119-2127

22. Bezu L, Keep O, Cerrato G et al (2018) Trial watch: peptide-based vaccines in anticancer therapy. OncoImmunology 7:e1511506

23. Zitvogel L, Apetoh L, Ghirnghelli F, Kroemer G (2008) Immunological aspects of anticancer chemotherapy. Nat Rev Immunol 8:59-73

24. Chan OT, Yang LX (2000) The immunological effects of taxanes. Cancer Immunol Immunother 49:181-185

25. Mason K, Staab A, Hunter N et al (2001) Enhancement of tumor radioresponse by docetaxel: involvement of immune system. Int J Oncol 18:599-606

26. Prell RA, Gearin L, Simmons A et al (2006) The anti-tumor efficacy of GM-CSF-secreting tumor cell vaccine is not inhibited by docetaxel administration. Cancer Immunol Immunother 55:1285-1293

27. Arlen PM, Gulley JL, Parker C et al (2006) A randomized phase II study of concurrent docetaxel plus vaccine versus vaccine alone in metastatic androgen-independent prostate cancer. Clin Cancer Res 12:1260-1269

28. Kongsted P, Borch TH, Ellebaek E et al (2017) Dendritic cell vaccination in combination with docetaxel for patients with metastatic castration-resistant prostate cancer: a randomized phase II study. Cytotherapy 19:500-513

29. Antonia SJ, Mirza N, Fricke I et al (2006) Combination of p53 cancer vaccine with chemotherapy in patients with extensive stage small cell lung cancer. Clin Cancer Res 12:878-887

30. Drake CG (2009) Immunotherapy for prostate cancer: walk, don't run. J Clin Oncol 27:4035-4037

31. Naito M, Itoh K, Komatsu N et al (2008) Dexamethasone did not suppress cancer patients. Prostate 68:1753-1762

32. Yoshimura K, Minami T, Nozawa M et al (2016) A phase 2 randomized controlled trial of personalized peptide vaccine immunotherapy with low-dose dexamethasone versus dexamethasone alone in chemotherapy-naïve castration-resistant prostate cancer. Eur Urol 70:35-41

33. Vuk-Pavlovic S, Bulur PA, Lin Y et al (2010) Immunosuppressive $\mathrm{CD} 14^{+} \mathrm{HLA}^{-} \mathrm{DR}^{\text {low/- }}$ monocytes in prostate cancer. Prostate 70:443-455

34. Liechtenstein T, Perez-Janices N, Gato M et al (2014) A highly efficient tumor-infiltrating MDSC differentiation system for discovery of anti-neoplastic targets, which circumvents the need for tumor establishment in mice. Oncotarget 5:7843-7857

Publisher's Note Springer Nature remains neutral with regard to jurisdictional claims in published maps and institutional affiliations. 


\section{Affiliations}

\section{Masanori Noguchi ${ }^{1,2}$ (1) $\cdot$ Gaku Arai $^{5} \cdot$ Shin Egawa ${ }^{6} \cdot$ Chikara Ohyama $^{7}$. Seiji Naito ${ }^{8} \cdot$ Kazumasa Matsumoto $^{9}$. Hirotsugu Uemura ${ }^{10}$. Masayuki Nakagawa ${ }^{11}$. Yasutomo Nasu ${ }^{12}$. Masatoshi Eto ${ }^{13}$. Shigetaka Suekane ${ }^{2}$. Tetsuro Sasada ${ }^{14} \cdot$ Shigeki Shichijo $^{1} \cdot$ Akira Yamada $^{3} \cdot$ Tatsuyuki Kakuma $^{4} \cdot$ Kyogo Itoh $^{1}$}

1 Canver Vaccine Center, Kurume University School of Medicine, 67 Asahi-machi, Kurume 830-0011, Japan

2 Department of Urology, Kurume University School of Medicine, 67 Asahi-machi, Kurume 830-0011, Japan

3 Cancer Vaccines, Research Center for Innovative Cancer Therapy, Kurume University School of Medicine, Kurume, Japan

4 Biostatistics Center, Kurume University School of Medicine, Kurume, Japan

5 Department of Urology, Dokkyo Medical University Koshigaya Hospital, Koshigaya, Japan

6 Department of Urology, Jikei University School of Medicine, Tokyo, Japan

7 Department of Urology, Graduate School of Medicine and School of Medicine, Hirosaki University, Hirosaki, Japan
8 Sanshinkai Hara Hospital, Fukuoka, Japan

9 Department of Urology, Kitasato University School of Medicine, Kanagawa, Japan

10 Department of Urology, Kinki University Faculty of Medicine, Osaka, Japan

11 Department of Urology, Kagoshima University Graduate School of Medical and Dental Science, Kagoshima, Japan

12 Department of Urology, Okayama University Graduate School of Medicine, Okayama, Japan

13 Department of Urology, Graduate School of Medical Sciences, University of Kyushu, Fukuoka, Japan

14 Kanagawa Cancer Center Research Institute, Kanagawa, Japan 